\title{
Signatures of nonlocal Cooper-pair transport and of a singlet-triplet transition in the critical current of a double-quantum-dot Josephson junction
}

\author{
B. Probst,${ }^{1}$ F. Domínguez, ${ }^{2,3}$ A. Schroer, ${ }^{1}$ A. Levy Yeyati, ${ }^{2}$ and P. Recher ${ }^{1,4}$ \\ ${ }^{1}$ Institut für Mathematische Physik, Technische Universität Braunschweig, D-38106 Braunschweig, Germany \\ ${ }^{2}$ Departamento de Física Teórica de la Materia Condensada, Condensed Matter Physics Center (IFIMAC), \\ and Instituto Nicolás Cabrera, Universidad Autónoma de Madrid, E-28049 Madrid, Spain \\ ${ }^{3}$ Institut für Theoretische Physik und Astrophysik, Universität Würzburg, D-97074 Würzburg, Germany \\ ${ }^{4}$ Laboratory for Emerging Nanometrology Braunschweig, D-38106 Braunschweig, Germany
}

(Dated: September 27, 2018)

\begin{abstract}
We study the critical Josephson current flowing through a double quantum dot weakly coupled to two superconducting leads. We use analytical as well as numerical methods to investigate this setup in the limit of small and large bandwidth leads in all possible charging states, where we account for on-site interactions exactly. Our results provide clear signatures of nonlocal spin-entangled pairs, which support interpretations of recent experiments [Deacon, R. S. et al., Nat. Commun. 6, 7446 (2015)]. In addition, we find that the ground state with one electron on each quantum dot can undergo a tunable singlet-triplet phase transition in the regime where the superconducting gap in the leads is not too large, which gives rise to an additional new signature of nonlocal Cooper pair transport.
\end{abstract}

PACS numbers: 74.50.+r,74.45.+c,03.65.Ud

\section{INTRODUCTION}

Creating mobile spin-entangled electron pairs in solid state transport setups has been the subject of intensive research in recent years 11 The proposed setup of Ref. 1 consists of an $s$-wave superconductor coupled to two quantum dots (QDs) in the Coulomb-blockade regime, which are further coupled to outgoing Fermi liquid leads,,$\frac{12}{16}$ where a dominant transport channel comprising pairwise and nonlocal transport has been identified. The degree of spin-entanglement of the nonlocal pairs, however, has so far not been measured as it is not straight forward to measure spin correlations directly. Many ways have been proposed to detect the spin entanglement ranging from violating a Bell inequality, 17,19 noise properties in a beam-splitter setup $20 \sqrt{25}$ or converting the electron-spin pairs to photons. ${ }^{26}+30$ Recently, Deacon et al ${ }^{31}$ realized a Josephson junction containing a double quantum dot (DQD) embedded in the junction. The critical current of the junction was investigated and signs of nonlocal pair transport coherently involving both QDs were conjectured by showing that the results are incompatible with two uncorrelated transport channels of supercurrent separately via each QD.

Here, we analyze in detail the setup of Ref. 31 using both analytical and numerical tools. We thereby go beyond existing theoretical work on the proposed setup 32133 by taking into account all of the filling factors of the QDs up to two levels per QD. We also relax the assumption of a large superconducting gap compared to the QD level energies $\varepsilon_{i}$ and the charging energy $U_{i}$ of the QDs $i=1,2$. The signatures of nonlocal pair transport we investigate do not rely on the Aharanov-Bohm effect 3 or on SQUIDs. $32[33$

We first introduce the model Hamiltonian in Sec. II and a special limit, the zero-bandwidth approximation, where the superconducting leads are represented by a single site with pairing interaction. We then calculate the critical current in the ground state of the junction numerically by exact diagonalization and compare it to a fourth-order perturbation theory in the tunneling from the DQD to the superconducting leads, both in the zero-bandwidth limit and in the wide-band limit. In the perturbation theory, the different tunneling paths, local and nonlocal contributions, become explicit. The ground state in the $(1,1)$-charging sector of the DQD (Figs. 1 and 3) changes as a function of the level energies on the QDs and/or charging energy from nonlocal singlet to nonlocal triplet. The triplet ground state is stabilized in the regime $\varepsilon_{i} /\left|\Delta_{\nu}\right|>1$ by cotunneling processes, which are independent of the superconducting phase difference and therefore are not of the Andreev reflection type. We show that the so-far undiscovered triplet phase also crucially depends on the parity of the tunneling matrix elements (Figs. 1 and 3). Going beyond the zero-bandwidth approximation by considering superconducting leads in the wide-band limit, we show perturbatively in the tunneling, that the triplet phase also exists in this case if the charging energies $U_{i}$ of the QDs are finite. This extends the model considered in Refs. 32 and 33 , where only singlet phases in the ground state are predicted, to the case of finite $U_{i}$ hosting both singlet and triplet ground states.

We further study a multilevel model numerically in which one of the QDs has an additional single-particle level, and show that this configuration gives a qualitatively good description of the experimental results of Ref. 31, assuming different parities for the tunneling matrix elements for the two levels on one QD.

Finally, we present the analytical case of large superconducting gaps, where we can integrate out the superconducting leads obtaining an effective model for the DQD system only. This parameter regime corresponds 


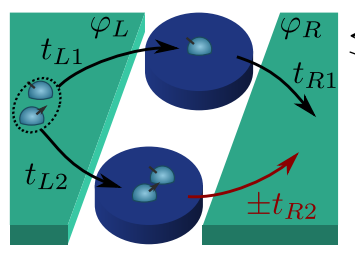

(a) (b)
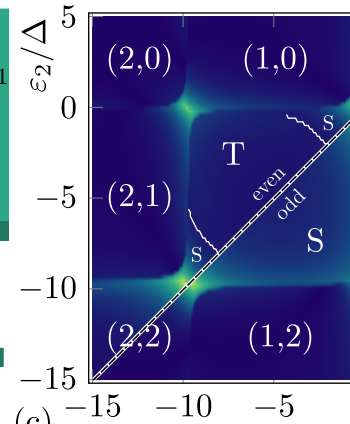

(c)

(c) -1
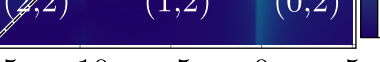

$\varepsilon_{1} / \Delta^{5}$

FIG. 1. (Color online) Double-quantum-dot Josephson junction. (a) The Josephson current is carried by Cooper pairs which tunnel coherently between two superconducting leads with superconducting phases $\varphi_{L}$ and $\varphi_{R}$. Microscopically, this involves four single-particle tunneling events with amplitudes $t_{\nu i}$. Local transport (both electrons of a Cooper pair tunnel through a single quantum dot) can be distinguished from nonlocal transport (the two electrons of a Cooper pair tunnel through different quantum dots). (b) The symmetry of the orbital wave functions on the quantum dots is captured in the total tunnel parity $\mathcal{P}=\operatorname{sgn}\left(t_{L 1} t_{L 2} t_{R 1} t_{R 2}\right)$, or, equivalently, in $\pm t_{R 2}$, and has distinctive signatures in the critical current. (c) Critical current across the junction at zero temperature as a function of the quantum-dot level energies $\varepsilon_{1,2}$ obtained in the zero-bandwidth approximation (Sec. II A). The upper-left half of the plot shows the critical current at even tunnel parity and the lower-right half at odd tunnel parity. The critical current becomes large close to ground-state transitions where the charge of the quantum dots $\left(N_{1}, N_{2}\right)$ fluctuates. At even tunnel parity a transition between a nonlocal singlet (S) and a triplet $(\mathrm{T})$ ground state in the $(1,1)$ sector emerges. The parameters are $|t|=0.5 \Delta$ and the Coulomb repulsion $U=10 \Delta$, where $\Delta$ is the magnitude of the superconducting gap in the leads.

to the limit where the nonlocal processes can be maximized by having, in addition, a charging energy much larger than the induced superconducting gap. Here, as expected, we obtain only a singlet ground state in the $(1,1)$ sector.

\section{MODEL}

We consider the geometry depicted in Fig. 1(a). Two QDs $i=1,2$ are tunnel coupled in parallel to two $s$-wave superconductors $\nu=L, R$ at $\mathbf{x}=0$ with amplitudes $t_{\nu i}$, which are chosen real in the absence of a magnetic field. Each QD contains only a single spin-degenerate level, $\sigma=\uparrow, \downarrow$, with energy $\varepsilon_{i}$ and with the local Coulomb repulsion $U_{i}$, which is relevant for transport. There is no direct cross-talk between the QDs or between the superconductors. The Hamiltonian is

$$
H=H_{1}+H_{2}+H_{L}+H_{R}+H_{T}
$$

with the QD contributions

$$
H_{i}=\sum_{\sigma} \varepsilon_{i} d_{i \sigma}^{\dagger} d_{i \sigma}+U_{i} d_{i \uparrow}^{\dagger} d_{i \downarrow}^{\dagger} d_{i \downarrow} d_{i \uparrow},
$$

the superconducting lead contributions

$$
H_{\nu}=\sum_{\mathbf{k} \sigma} \varepsilon_{\mathbf{k} \nu} c_{\mathbf{k} \nu \sigma}^{\dagger} c_{\mathbf{k} \nu \sigma}+\sum_{\mathbf{k}} \Delta e^{-i \varphi_{\nu}} c_{\mathbf{k} \nu \uparrow}^{\dagger} c_{-\mathbf{k} \nu \downarrow}^{\dagger}+\text { H.c. }
$$

and their tunnel coupling

$$
\begin{aligned}
H_{T} & =\sum_{i \sigma \nu} t_{\nu i} d_{i \sigma}^{\dagger} \psi_{\nu \sigma}(0)+\text { H.c. } \\
& =\sum_{i \mathbf{k} \sigma \nu} t_{\nu i} d_{i \sigma}^{\dagger} c_{\nu \mathbf{k} \sigma}+\text { H.c. }
\end{aligned}
$$

where the $d_{i \sigma}$ operators annihilate electrons localized on the QDs and where the $c_{\mathbf{k} \nu \sigma}$ and the $\psi_{\nu \sigma}(\mathbf{x})$ operators annihilate spin- $\sigma$ electrons in lead $\nu$ with momentum $\mathbf{k}$ or at position $\mathbf{x}$, respectively. The normal-state dispersion in the leads is $\varepsilon_{\mathbf{k} \nu}$. We assume the two superconducting leads to be of the same material with the same superconducting energy gap $\Delta$. Their superconducting phases $\varphi_{\nu}$ are not equal if a finite supercurrent flows across the DQD structure but only the difference between the superconducting phases, $\Delta \varphi=\varphi_{L}-\varphi_{R}$, is a gauge-invariant quantity, which enters the physical observables, and is conveniently absorbed into the tunnel couplings to the right lead, whereas the couplings to the left lead are strictly real. Furthermore, the behavior of the Hamiltonian, Eq. (1), depends drastically on the sign of the tunnel couplings. This sign is determined by the overlap between the wavefunctions in the respective superconducting lead and on the QD. Having assumed that the leads are $s$-wave superconductors, the sign depends on the orbital parity of the QD levels, i.e., for an even orbital both the left and right tunnel coupling have the same sign, while they have opposite signs for an odd orbital [Fig. 1(b)]. We can recast all the possible sign combinations into two possibilities by defining the total tunnel parity, ${ }^{\sqrt[36]{ }} \mathcal{P}=\operatorname{sgn}\left(t_{L 1} t_{L 2} t_{R 1} t_{R 2}\right)$, which may be gauged arbitrarily into one of the tunnel couplings. Accounting for both of the effects, we replace $t_{R 1} \rightarrow t_{R 1} e^{i \Delta \varphi / 2}$ and $t_{R 2} \rightarrow \mathcal{P} t_{R 2} e^{i \Delta \varphi / 2}$, where all $t_{\nu i}>0$.

We will discuss the case in which both QDs are in the single-level and Coulomb-blockade regime where the level broadening due to the tunnel couplings to the leads, $\Gamma_{\nu i}=2 \pi N\left(\varepsilon_{F}\right)\left|t_{\nu i}\right|^{2}$ with $N\left(\varepsilon_{F}\right)$ the normal-state density of states at the Fermi level, is much smaller than the level spacing and than the Coulomb repulsion, $\Gamma_{\nu i} \ll \delta \varepsilon_{i}, U_{i}$. Then the QDs have a well-defined charge $\left(N_{1}, N_{2}\right)$ except close to transport resonances [Fig. 11(c)].

In the presence of interactions, there are several approaches that have been used to study transport through a DQD Josephson junction, including mean field $\stackrel{37}{, 3}$ slave-boson mean field, $\stackrel{38 \mid 39}{r}$ renormalization group methods, ${ }^{40 \mid 41}$ real-time diagrammatic expansion,, 42 quantum Monte Carlo $\stackrel{43}{,}$ and finite order perturbation 
theory. ${ }^{44}$ Here we combine a zero-bandwidth approximation and conventional perturbation theory to obtain both reliable results and physical insight. We restrict this analysis to the critical Josephson current $I_{c}$ at low temperatures $T$ by setting $T=0$. Even though this is a macroscopic and thus directly accessible quantity, we will see that it contains various clear signatures of nonlocal Cooper-pair transport, some of which have already been observed ${ }^{31}$ Having obtained the ground-state energy $E_{0}(\Delta \varphi)$ of $H$ either by perturbation theory or by exact diagonalization, the critical current is immediately given as the maximum supercurrent

$$
I_{c}=\frac{2 e}{\hbar} \max _{\Delta \varphi} \frac{\partial E_{0}(\Delta \varphi)}{\partial \Delta \varphi}
$$

supported by the ground state of the system.

\section{A. Zero-bandwidth approximation}

We follow Ref. 45 and integrate out the superconducting leads, yielding the effective lead Hamiltonian

$$
H_{\nu}^{\mathrm{zbw}}=\Delta_{b} c_{\nu \uparrow}^{\dagger} c_{\nu \downarrow}^{\dagger}+\text { H.c. }
$$

and renormalized tunnel parameters $t_{\nu i} \rightarrow \tilde{t}_{\nu i}^{b}$. Thus, the Josephson junction is represented by a four-site superconducting molecule, which can be exactly diagonalized. Former studies using the zero-bandwidth approach in interacting Josephson junctions have proven to show qualitatively good agreement with mean-field calculations. This approach retains the essential features of the competition between pairing correlations and Kondo correlations occurring in the single-QD case $\frac{46 / 47}{}$ The renormalized parameters $\Delta_{b}, t_{\nu i}^{b}$ may be obtained from selfconsistent calculations. ${ }^{45}$ We compare the results coming from the zero-bandwidth lead Hamiltonian, Eq. (6), with the fourth-order perturbation-theory calculations using the original Hamiltonian, Eq. (3), and find very good agreement already using the bare couplings, $\Delta_{b}=\Delta$ and $t_{\mu i}^{b}$, in the regime where $t_{\nu i} \lesssim|\Delta|$, up to a global prefactor, which is proportional to the energy density of states of the normal-state leads.

\section{B. Perturbation theory and microscopic behavior}

The numerical treatment by exact diagonalization has to be accompanied by a perturbative treatment to identify the physical processes giving rise to the critical current, in particular, to understand which features are attributable to nonlocal Cooper-pair transport. Furthermore perturbation theory is not restricted to the zerobandwidth approximation. We calculate the corrections to the ground-state energy of the isolated QDs due to their coupling to the superconducting leads in fourth order of $t_{i \nu}$, which is the leading order of Cooper-pair transfer between the leads. To handle the large amount of processes available in fourth order we develop a diagrammatic scheme, the details of which are given in App. A

In the unperturbed ground state, there are no excitations in the leads and the QDs have a well-defined charging state $\left(N_{1}, N_{2}\right)$, where $N_{i}$ is the number of electrons on QD $i$. Each charging state is spin degenerate and can be realized by different quantum states, $|\alpha, \beta\rangle:=|\alpha\rangle_{\mathrm{QD} 1}|\beta\rangle_{\mathrm{QD} 2}$, all of which are completely decoupled because the model conserves the $z$ projection of the total spin, $S_{z}$. The only exception are the states in the $(1,1)$ sector with $S_{z}=0$, where degenerate perturbation theory in the space spanned by the states $|\uparrow, \downarrow\rangle$ and $|\downarrow, \uparrow\rangle$ is required. Since the total spin is conserved, the degenerate subspace is diagonal in the basis of the nonlocal singlet and the nonlocal triplet,

$$
\begin{aligned}
& |S\rangle=\frac{1}{\sqrt{2}}(|\uparrow, \downarrow\rangle-|\downarrow, \uparrow\rangle) \\
& |T\rangle=\frac{1}{\sqrt{2}}(|\uparrow, \downarrow\rangle+|\downarrow, \uparrow\rangle) .
\end{aligned}
$$

Spin-exchange processes, i.e., processes which swap the spins of the QDs, split the singlet and the triplet. Note that the spin triplets with $S_{z}= \pm 1$ behave equivalently to $|T\rangle$ by spin-rotation invariance.

The terms in the perturbative expansion are local or nonlocal, where local processes involve only one of the QDs whereas nonlocal processes involve both QDs. Furthermore, we call all processes Josephson processes, in which entire Cooper pairs are removed from or added to the superconducting leads due to two single-particle tunnel events. In processes which are not Josephson processes as many carriers are added to each lead as are removed, so we call them cotunneling processes $\stackrel{48}{\underline{4}}$

Summarizing all of the processes, we can write down the general form of the correction of the ground-state energy in perturbation theory,

$$
\begin{gathered}
\delta E_{0}(\Delta \varphi)=E_{\mathrm{CT}, 1}^{\mathrm{loc}}+E_{\mathrm{CT}, 2}^{\mathrm{loc}}+E_{J, 1}^{\mathrm{loc}}(\Delta \varphi)+E_{J, 2}^{\mathrm{loc}}(\Delta \varphi) \\
+E_{\mathrm{CT}, \mathrm{sc}}^{\mathrm{nl}}+E_{J, \mathrm{sc}}^{\mathrm{nl}}(\Delta \varphi) \pm\left[E_{\mathrm{CT}, \mathrm{se}}^{\mathrm{nl}}+E_{J, \mathrm{se}}^{\mathrm{nl}}(\Delta \varphi)\right]
\end{gathered}
$$

where the superscript denotes whether the correction is due to local (loc) or nonlocal (nl) processes and the subscripts denote whether the correction comes from a cotunneling $(\mathrm{CT})$ or from a Josephson $(\mathrm{J})$ process. For local processes, the second subscript denotes the QD which is involved in the process whereas for nonlocal processes, the second subscript denotes whether the process is a spin-exchanging (se) or spin-conserving (sc) process. The spin-exchange contributions are nonzero only in the $(1,1)$ sector. In the $(1,1)$ sector, Eq. (9) is thus the energy correction of the nonlocal triplet (upper sign) and the nonlocal singlet (lower sign).

In the charge sectors with a unique ground state, the critical current is given directly by the amplitude of the phase-dependent corrections of the ground-state energy, which, in perturbation theory, are proportional to $\cos (\Delta \varphi)$. The amplitude is commonly referred to as 


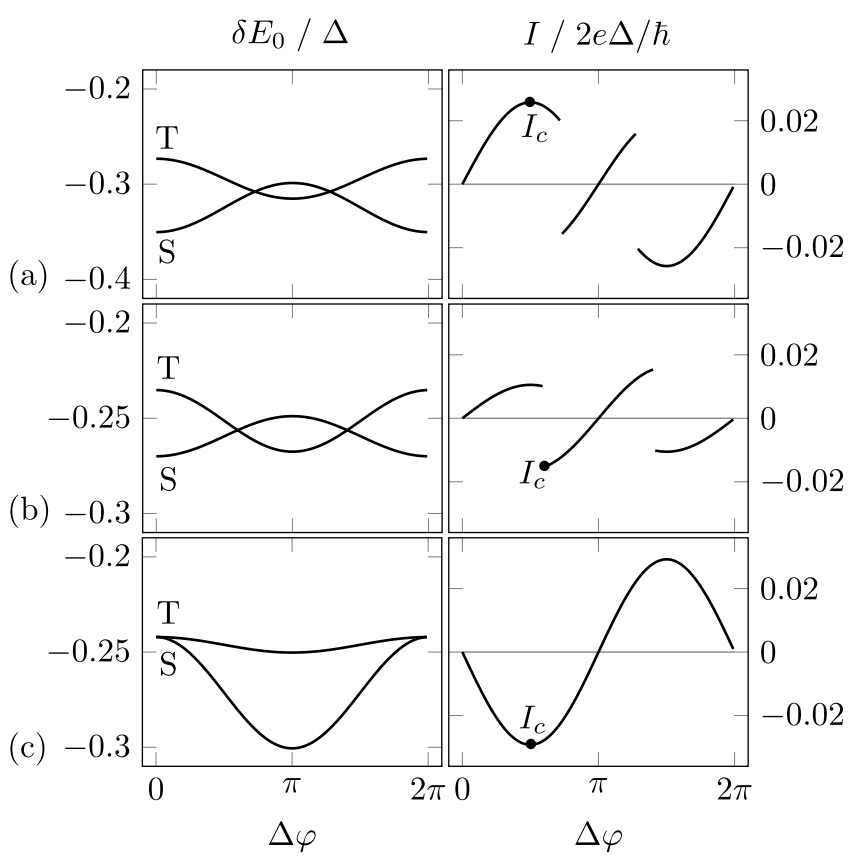

FIG. 2. Phase dependence of the energy corrections $\delta E_{n}$ of the two lowest-lying states in the $(1,1)$-charge sector and the resulting supercurrent $I$ in the ground state evaluated in the zero-bandwidth approximation. (a) Point A in Fig. 3 . The critical current $I_{c}$ is carried by the singlet ground state $(\mathrm{S})$ at $\Delta \varphi= \pm \pi / 2$. (b) Point B in Fig. 3. The critical current is carried by the triplet ground state (T) at $\Delta \varphi \neq \pm \pi / 2$. (c) Point $\mathrm{C}$ in Fig. 3. At odd total tunnel parity, $\mathcal{P}=-1$, the ground state is a singlet at all $\Delta \varphi$ and the critical current has the conventional sinusoidal dependence on $\Delta \varphi$. The junction is in the $\pi$ phase.

the (phase-independent) Josephson energy $E_{J}$ and the critical current is proportional to the Josephson energy, $I_{c} \propto E_{J}$. Since $E_{J}$ decomposes into local and nonlocal contributions, so does $I_{c}$. The critical phase is always at $\Delta \varphi= \pm \pi / 2$, where $\partial_{\Delta \varphi} \cos (\Delta \varphi)$ is maximized.

In the $(1,1)$-charge sector, the situation is more complicated. Both the energy of the singlet state and the energy of the triplet state, cf. Eq. (9), depend on the phase difference such that they may cross for suitable parameters and hence the ground state changes between singlet and triplet as a function of the phase difference $\Delta \varphi$. Three possible situations are shown in Fig. 2 If there is a singlet-triplet ground-state transition as a function of the phase difference, the cosinelike energy-phase relation of the ground state $\delta E_{0}(\Delta \varphi)$ becomes a piecewise function of the phase difference with two different amplitudes and with two different constant energy offsets for the singlet state and the triplet state, cf. right panels of Figs. 2(a) and (b). When the critical current is probed, the junction adjusts to the phase difference which maximizes the supercurrent. This is not necessarily at the conventional value $\Delta \varphi= \pm \pi / 2$. At $\Delta \varphi= \pm \pi / 2$ there might be a singlet (triplet) ground state with a low amplitude $E_{J}(\Delta \varphi)$ which cannot carry as high a supercurrent as the triplet (singlet) ground state at a different phase difference $\Delta \varphi^{\prime} \neq \pm \pi / 2$ but with a larger amplitude $E_{J}\left(\Delta \varphi^{\prime}\right)$ such that $\left|E_{J}( \pm \pi / 2)\right|<\left|E_{J}\left(\Delta \varphi^{\prime}\right) \sin \left(\Delta \varphi^{\prime}\right)\right|$ [Fig. 2(b)] ${ }^{49}$ Then the junction will switch to the triplet (singlet) ground state and the critical phase locks to $\Delta \varphi^{\prime}$.

\section{RESULTS}

\section{A. Singlet-triplet ground-state transition}

In Fig. 3, we present the results for the critical current of the DQD Josephson junction. In Fig. 3(a), we plot the critical current and the total spin of the QD system in the ground state carrying the critical current depending on $U=U_{1}=U_{2}$ and on $\varepsilon_{1}$ at fixed $\varepsilon_{2}=-1.5 \Delta$ in the zerobandwidth limit. When the tunnel parity is even, $\mathcal{P}=1$, (left panels) the total spin in the $(1,1)$ sector changes from a singlet to a triplet in a regime of finite charging energy $U$. This is true both in the zero-bandwidth limit (solid and dash-dotted phase boundaries) and in the wide-band limit (dashed phase boundary) with no qualitative differences. In the wide-band limit, the normalstate density of states in the superconducting leads is constant at all relevant QD energies. Then it affects the critical current only as a constant prefactor, which we choose to fit the zero-bandwidth results. With the tunnel couplings $\left|t_{\nu i}\right|=0.5 \Delta$, as chosen in Fig. 3 , and a superconducting gap on the order of $\Delta=0.1 \mathrm{meV}$, we obtain a critical current of a few nanoampere at the resonances. This agrees with the experimental data of Ref. 31, where aluminum electrodes were used 31150

In the wide-band limit at very large Coulomb repulsion, $U_{i} \rightarrow \infty$, the parameter space is confined to what is the upper right corner of the $(1,1)$ sector in Fig. 11(c) and the triplet ground state cannot be observed, consistent with earlier studies ${ }^{32}$ Numerically, we confirm that the triplet ground state can emerge if either the Coulomb repulsion or the bandwidth are not significantly larger than all other energy scales, which makes it rather the rule than the exception.

Intuitively, one could expect a singlet ground state in the $(1,1)$-charge sector, which could be justified by noting that only the nonlocal singlet can tunnel into the leads to hybridize with the Cooper pairs in the $s$-wave superconductors and lower its energy. However, in addition to this second-order Cooper-pair tunneling process, there are genuine fourth-order terms with additional intermediate single-particle excitations in the superconducting leads. They cannot be decomposed into two Cooper-pair tunnel events and may favor the triplet ground state.

The splitting of the nonlocal singlet and the nonlocal triplet is given by $E_{C T, s e}^{\mathrm{nl}}+E_{J, s e}^{\mathrm{nl}}(\Delta \varphi)$. Since the superconductors are identical, the energy corrections can be split into a matrix element $\mathcal{M}_{\mathrm{CT} / \mathrm{J}, \mathrm{se} / \mathrm{sc}}^{\mathrm{nl} / \mathrm{gc}}$ and the tunnel couplings to the leads. Since we associate the parity $\mathcal{P}$ and the phase difference $\Delta \varphi$ to the tunnel couplings $t_{\nu i}$, 

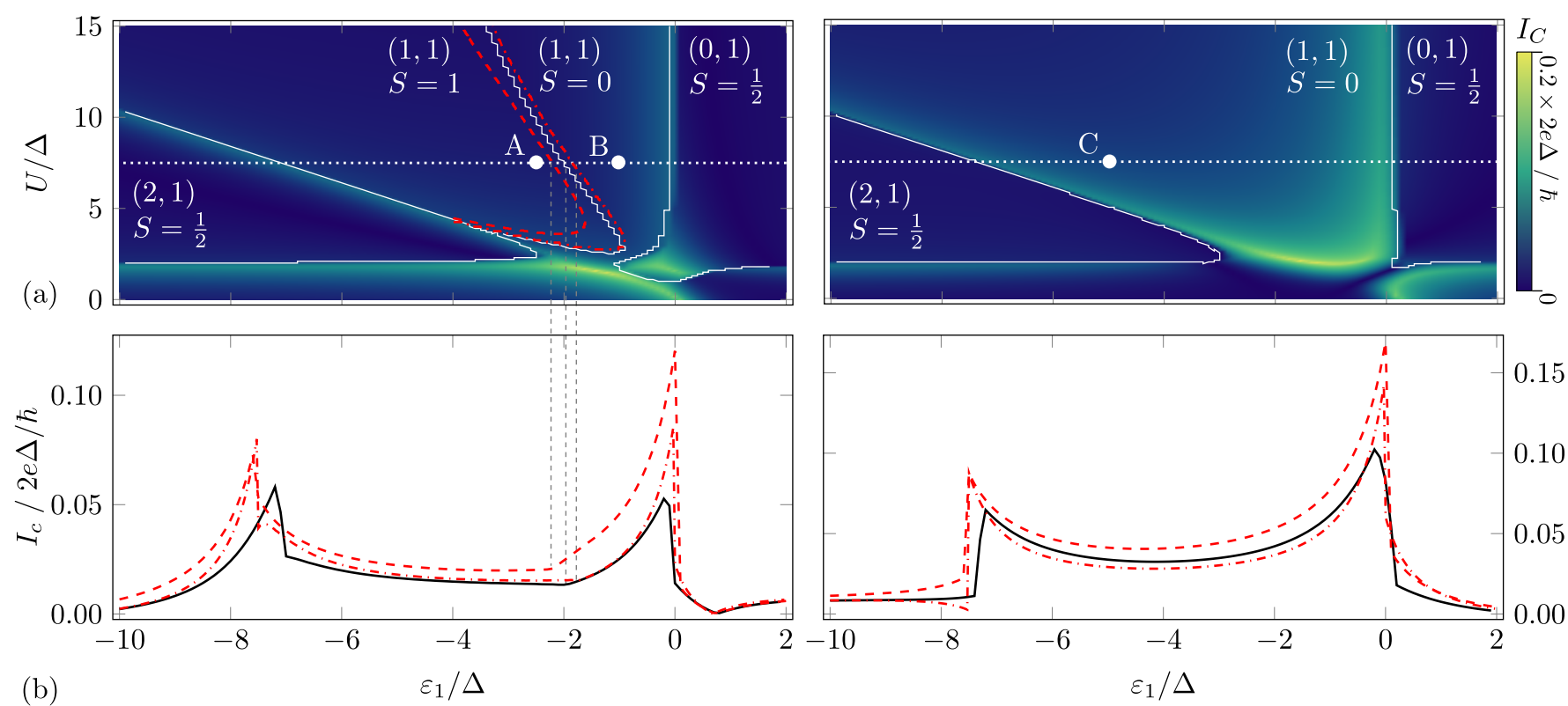

FIG. 3. (Color online) (a) Critical current and total spin of the Josephson junction depending on the on-site energy $\varepsilon_{1}$ on quantum dot 1 and on the Coulomb repulsion $U=U_{1}=U_{2}$. Quantum dot 2 is kept at $\varepsilon_{2}=-1.5 \Delta$ and the tunnel couplings are $\left|t_{\nu i}\right|=0.5 \Delta$. Following the white dotted line from left to right, the quantum-dot occupation varies $(2,1) \rightarrow(1,1) \rightarrow(0,1)$. The critical current increases at each transition because the particle number fluctuates. Left: at even total tunnel parity, $\mathcal{P}=1$, an additional ground-state transition between a nonlocal singlet and a nonlocal triplet occurs in the $(1,1)$ sector. It is caused by competing cotunneling processes between the quantum dots via the superconducting leads which give rise to an exchange interaction (text). The red lines indicate the phase boundary obtained in perturbation theory in the zero-bandwidth approximation (dash dotted) and in the wide-band limit (dashed). Right: at odd total tunnel parity, $\mathcal{P}=-1$, the singlet-triplet transition is absent. (b) Cuts across the $\varepsilon_{1}-U$ plane at $U=7.5 \Delta$ reveal that the shape of the current peaks depends strongly on the tunnel parity. This can be traced back to the singlet-triplet transition (text), which also immediately manifests as a kink in the critical current. Since singlet and triplet can be distinguished only by nonlocal transport, this kink is immediate evidence of coherently split Cooper pairs. There is no qualitative difference between the zero-bandwidth approximation (exact: solid black, perturbative: dash-dotted red) and the wide-band limit (dashed red). In the wide-band limit, the critical current scales with the density of states, which is chosen to agree with the result in zero-bandwidth approximation.

the matrix element is then independent of either. Each nonlocal process contributing to $E_{J, \mathrm{se}}^{\mathrm{nl}}(\Delta \varphi)$ can involve the same superconducting lead twice or both leads once. Summing all combinations we obtain

$$
\begin{aligned}
E_{J, \mathrm{se}}^{\mathrm{nl}}(\Delta \varphi)= & \mathcal{M}_{J, \mathrm{se}}^{\mathrm{nl}} \\
& \times\left[\left(t_{R 1} t_{R 2}\right)^{2}+\left(t_{L 1} t_{L 2}\right)^{2}\right. \\
& \left.\quad+2 \mathcal{P} t_{R 1} t_{R 2} t_{L 1} t_{L 2} \cos (\Delta \varphi)\right],
\end{aligned}
$$

where we find $\mathcal{M}_{J, \mathrm{se}}^{\mathrm{nl}}>0$, such that $E_{J, \mathrm{se}}^{\mathrm{nl}}$ is strictly positive and favors the singlet ground state. The details of the calculation of the matrix elements are given in App. A.

With the same arguments, we find

$$
\begin{aligned}
& E_{\mathrm{CT}, \mathrm{se}}^{\mathrm{nl}}=\mathcal{M}_{\mathrm{CT}, \mathrm{se}}^{\mathrm{nl}} \\
& \times\left[\left(t_{R 1} t_{R 2}\right)^{2}+\left(t_{L 1} t_{L 2}\right)^{2}+2 \mathcal{P} t_{R 1} t_{R 2} t_{L 1} t_{L 2}\right] \\
& =\mathcal{M}_{\mathrm{CT}, \mathrm{se}}^{\mathrm{nl}}\left(t_{R 1} t_{R 2}+\mathcal{P} t_{L 1} t_{L 2}\right)^{2},
\end{aligned}
$$

so the sign of $E_{\mathrm{CT}, \mathrm{se}}^{\mathrm{nl}}$ is determined solely by $\mathcal{M}_{\mathrm{CT}, \mathrm{se}}^{\mathrm{nl}}$. This time, however, the perturbative analysis of $\mathcal{M}_{\mathrm{CT} \text {, se }}^{\mathrm{nl}}$ reveals that processes of both signs exist. Processes in which the electrons are exchanged via electronlike excitations in the leads [Fig. 4(a)] have a different number of fermion-exchange signs than processes in which the electrons are exchanged via an electronlike and a holelike excitation [Fig. 4(b)]. Processes involving only electronlike excitations lower the singlet while process involving an electronlike and a holelike excitation lower the triplet. All spin-exchange processes are listed in App. A.

For the existence of a triplet ground state the Josephson processes play a minor role as they are suppressed if $\Delta \varphi$ is chosen such that $\mathcal{P} \cos \Delta \varphi=-1$. The nonlocal triplet is thus driven by the sign of $E_{\mathrm{CT} \text {, se }}^{\mathrm{nl}}$, which is ultimately determined by the microscopic parameters.

The influence of $\Delta$ on the parameter space in which there may be a triplet ground state can be estimated. The matrix element of each process in the perturbative expansion is weighted by the product of the reciprocal virtual excitation energies (cf. App. A). In electronlike 

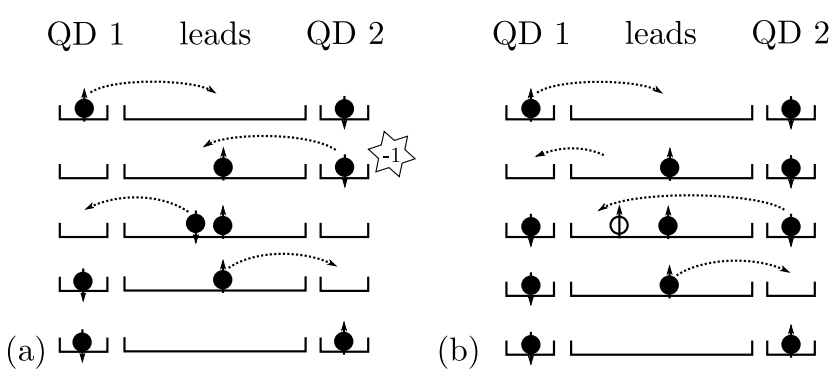

FIG. 4. Two spin-exchange processes which have a different overall sign and hence energetically favor (a) singlet states and (b) triplet states. Initially, one of two electrons (filled circles) with opposite spin resides on each quantum dot (left and right narrow tray). A final state with the spins swapped can be reached via intermediate virtual states (arranged top to bottom) connected by four tunnel processes (dashed arrows) between the quantum dots and the superconducting leads (wide tray). Every time the left-to-right order of two fermions is changed, a sign results. (a) If only electronlike states in the leads are involved, the two initial electrons have to be swapped. This kind of process with a negative sign energetically favors the singlet state. (b) If the exchange process involves a hole (empty circle), it is possible to exchange the spins without anticommutation signs. This type of process energetically favors the triplet state.

processes, which favor the singlet ground state, all virtual states involve excitations on the QDs. They can be estimated by

$$
\frac{1}{\left(\varepsilon_{\mathrm{DQD}}+\Delta\right)^{2}} \frac{1}{2 \varepsilon_{\mathrm{DQD}}+2 \Delta},
$$

where $\varepsilon_{\mathrm{DQD}}$ is a typical DQD-excitation energy. By using electronlike and holelike excitations, however, it is possible to restore the initial DQD state at the expense of two virtual excitations in the leads. These processes, which favor the triplet ground state, are hence weighted by

$$
\frac{1}{\left(\varepsilon_{\mathrm{DQD}}+\Delta\right)^{2}} \frac{1}{2 \Delta}
$$

If $\Delta$ is comparable or smaller than $\varepsilon_{\mathrm{DQD}}$, the ratio between triplet-favoring and singlet-favoring processes, $1+\varepsilon_{\mathrm{DQD}} / \Delta$, becomes large and a triplet ground state may emerge.

In general, second-order Cooper-pair tunneling restores the ground state of the superconducting leads in one intermediate virtual state, whereas the leads are excited in all three intermediate states of genuine fourthorder processes. So, genuine fourth-order processes have an additional suppression by $\Delta^{-1}$ compared to secondorder Cooper-pair processes. ${ }^{51}$ If the superconducting gap is very large compared to the other energy scales, the singlet character induced by the superconducting leads dominates and we recover the intuitive singlet ground state. We investigate this limit in more detail in Sec. V.

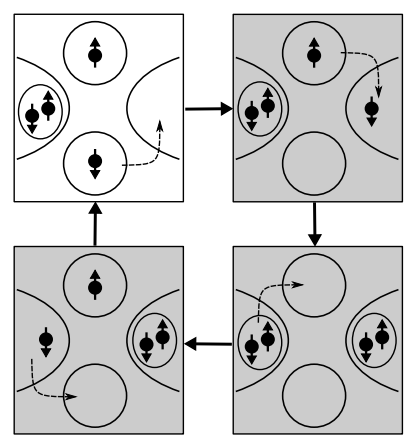

(a)

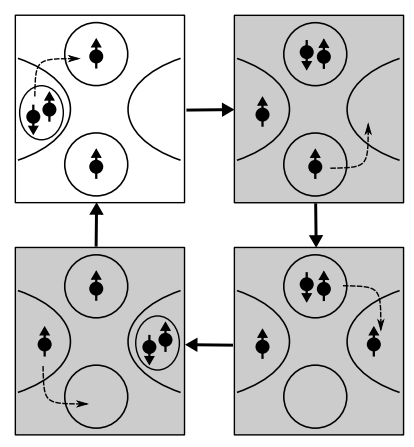

(b)
FIG. 5. Typical Josephson transport processes via three intermediate virtual states (gray) in the $(1,1)$-charge sector. (a) In the singlet ground state, there are transport channels in which the two electrons initially localized on the DQD are absorbed as a Cooper pair in one lead. (b) In the triplet ground state (all triplets are equivalent by spin-rotation invariance and time-reversal symmetry), the electrons of the Cooper pair need to be transferred sequentially through the double quantum dot.

The triplet ground state is absent in the regime of odd total tunnel parity, $\mathcal{P}=-1$, [Fig. 3(a), right panel]. This is because different cotunneling processes interfere destructively, which reduces the magnetic exchange coupling. At negative tunnel parity, $\mathcal{P}=-1$, the paritydependent factor in Eq. (11) is reduced and even vanishes in a symmetric setup, $t_{L i}=t_{R i}$. Without the exchange coupling, the nonlocal Josephson processes will always favor the singlet over the triplet, cf. Eq. (10) and Fig. 2(c).

\section{B. Peak asymmetry and signature of nonlocal transport}

Fig. 3(b) shows the critical current as a function of $\varepsilon_{1}$ for a fixed on-site repulsion $U=7.5 \Delta$ in the $\mathcal{P}= \pm 1$ regimes. Red lines are the results from perturbation theory in the zero-bandwidth limit (dash dotted) and in the wide-band limit (dashed), both of which agree with the exact results of the zero-bandwidth model (black solid). In general, the critical current is high at the charge neutrality points where the number of electrons on the QDs can fluctuate.

Both the singlet ground state and the triplet ground state can support a finite supercurrent. In the singlet phase, the supercurrent tends to be higher because there is an additional transport channel where the two electrons of a Cooper pair are simultaneously added to or removed from the DQD. In the triplet ground state, this channel is blocked by the Pauli exclusion principle. At the resonance near $\varepsilon_{1}=0$ in Fig. 3.(b), the QD charging states $(1,1),(1,0),(0,1)$, and $(0,0)$ are almost degenerate so this type of transport is particularly strong and in the singlet ground state the supercurrent is primarily carried by the process shown in Fig. 5(a). By inspecting 
all possible combinations, it is easy to see that there is no fourth-order transport process in the triplet ground state involving the $(0,0)$-charging state ${ }^{52}$ At the other resonance, however, $\varepsilon_{1} \approx-U_{1}$, a Josephson process involving the almost-degenerate QD states $(1,1),(1,0),(2,1)$, and $(2,0)$ does exist in the triplet ground state [Fig. 5(b)]. So with increasing $\varepsilon_{1}$ in Fig. 3(b), the singlet ground state has resonances both at the $(2,1)-(1,1)$ transition and at the $(1,1)-(0,1)$ transition but the triplet ground state has only one resonance at the $(2,1)-(1,1)$ transition. Hence, at even parity, with increasing $\varepsilon_{1}$ the critical current decreases in the $(1,1)$ sector as long as the system is still in the triplet ground state. Only once the ground state switches to a singlet, which happens close to the $(1,1)-(0,1)$ transition [cf. Fig. 3(a)], the critical current rises again, producing a notable asymmetry between the resonance peaks. At odd parity, there is no asymmetry because the ground state remains a singlet throughout the entire $(1,1)$ sector.

We emphasize that the singlet-triplet transition of the ground state in the $(1,1)$ sector, realized in a large parameter window, leads to a kink in the critical current as a function of $\varepsilon_{2}$. This kink appears because, in the singlet phase, different processes contribute to the critical current than in the triplet phase and, hence, the dependency on the on-site energies changes across the singlettriplet transition. Since the distinction between triplet and singlet phases results from phase coherent and nonlocal exchange, its observation in the critical current is a clear sign of nonlocal Cooper-pair transport.

At odd tunnel parity, $\mathcal{P}=-1$, there is no singlettriplet transition and hence no signature of nonlocal transport in the critical current. The other way around, if two neighboring resonance peaks belonging to the same level of a QD decay symmetrically in the offresonant regime between them, the level has odd parity. An asymmetric decay may be caused by a singlet-triplet transition and indicates even parity.

\section{MULTILEVEL QUANTUM DOT}

In order to make contact to the experiments presented in Ref. 31, where multiple QD levels were probed, we include one extra level in the model, e.g., on QD 2. In this way, we can study the evolution of the critical current along four consecutive resonances by continuously tuning $\varepsilon_{2}$. This scenario requires the substitution of $H_{2}$ in Eq. (1) by

$$
\begin{aligned}
H_{2}=\varepsilon_{2} d_{21 \sigma}^{\dagger} d_{21 \sigma}+\left(\varepsilon_{2}+\delta\right) & d_{22 \sigma}^{\dagger} d_{22 \sigma} \\
& +\sum_{(i \sigma) \neq(j \rho)} U_{i j} n_{2 i \sigma} n_{2 j \rho}
\end{aligned}
$$

where $\delta$ is the energy separation between the QD levels, and $U_{i j}$ the Coulomb energy coming from the interaction of the occupation of the levels $i$ and $j$ on the second

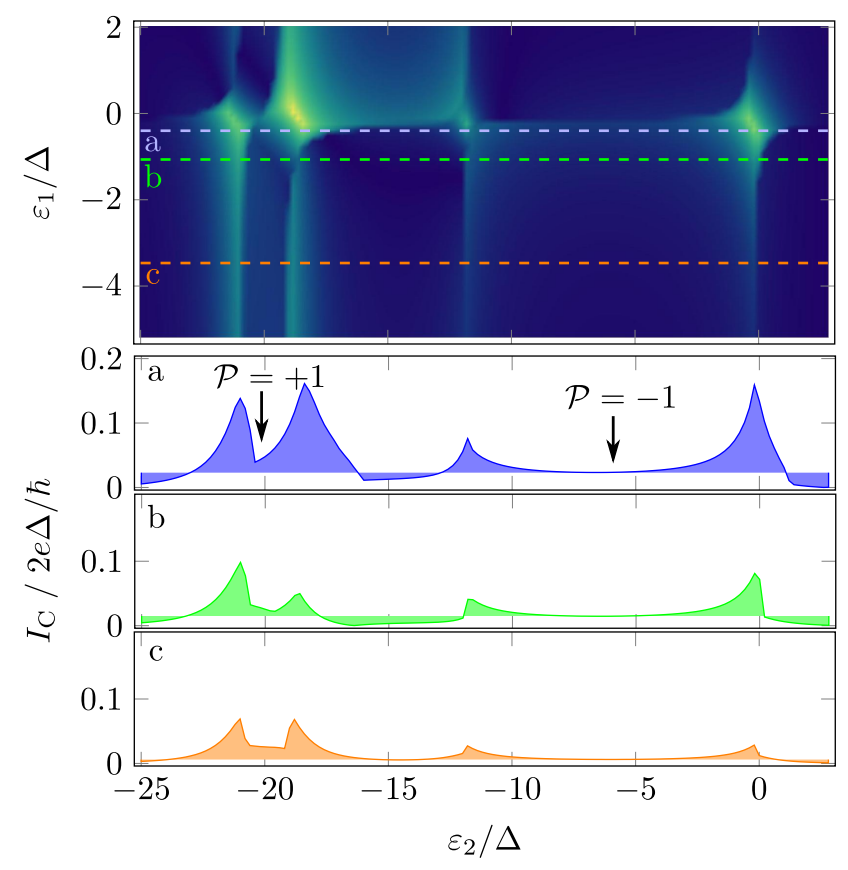

FIG. 6. (Color online) Critical current of the doublequantum-dot junction with two different levels of opposite parity on quantum dot 1 . The parameters of the quantum dots are $\delta=18.5 \Delta, t_{R 1}=t_{L 1}=0.45 \Delta, t_{R 21}=-t_{L 21}=$ $0.45 \Delta, t_{R 22}=t_{L 22}=0.57 \Delta, U_{1}=28 \Delta, U_{11}=12 \Delta$, $U_{22}=3 \Delta$, and $U_{12}=0.5 \Delta$. Upper panel: critical current as a function of the gate-controlled on-site energies $\varepsilon_{1}$ and $\varepsilon_{2}$. Lower panel: cuts at (a) $\varepsilon_{1}=-0.9 \Delta$, (b) $\varepsilon_{1}=-1.8 \Delta$, and (c) $\varepsilon_{1}=-4.2 \Delta$. In the absence of nonlocal transport, the three curves are expected to differ only by a constant. Instead, when approaching the resonance, $\varepsilon_{1} \rightarrow 0$, the critical current grows more strongly at the peaks and less strongly between the peaks. Within our model this behavior is clearly attributable to nonlocal coherent transport and it was already observed experimentally!

QD ${ }^{53}$ Besides, we also need to include an additional tunnel coupling to Eq. (4). Computationally, the addition of the extra level requires to extend the $256 \times 256$ Hamiltonian matrix to a $1024 \times 1024$ matrix, which remains tractable. Taking into account that the levels are well separated, we can still define the total tunnel parity close to a resonance as within the single-level model involving only the four relevant tunnel couplings.

Choosing the measurement presented in Fig. 4 of Ref. 31 as a specific example, we observe that two neighboring resonance peaks at lower gate voltages (higher onsite energies) are clearly more symmetric than two neighboring resonances at higher gate voltages (lower on-site energies). Within our model this is expected if the two lower peaks belong to one level with odd parity and the two higher peaks belong to one level with even parity (cf. Fig. 3). Note that concerning the occupation numbers this does not agree with Ref. 31, which seems to suggest that, in total, three levels on QD 2 are involved. 
Nevertheless, the model is clearly capable of reproducing the qualitative features observed in the experiment when choosing the appropriate parameters.

In Fig. 6 we show the critical current as a function of $\varepsilon_{2}$ and $\varepsilon_{1}$ (top panel) and in the lower panel we perform three cuts at different values of $\varepsilon_{1}$. Close to the resonance (blue and green curves), the results are basically equivalent to the results from the single-level model, once with even parity, and once with odd parity. Here, we recover the signature of nonlocal transport proposed in Ref.31; if there were only two independent transport channels, local transport through QD 1 and local transport through QD 2, the blue and the green curve would only differ from each other by being shifted along the vertical axis. This is because changing $\varepsilon_{1}$ would only affect the contribution of the critical current going through QD 1, which is independent from $\varepsilon_{2}$, i.e., it cannot influence the behavior of the critical current along the horizontal axis in the lower panel of Fig. 6. Choosing, however, an arbitrary reference point as indicated by the shaded areas, we can clearly see that there is cross-talk between $\varepsilon_{1}$ and $\varepsilon_{2}$. When QD 1 is brought closer to resonance, the resonance peaks of QD 2 grow, indicating an additional transport channel involving both QD 1 and QD 2. Moreover, there are interference effects which reduce the critical current between the two levels on QD 2, when QD 1 is brought closer to resonance. More strikingly, for values of $-\varepsilon_{1}>t, U$, we observe that the resonance at $\varepsilon_{2} \approx-18 \Delta$, increases when effectively decoupling QD 1 (yellow curve). Now, the Cooper pairs tunnel locally through QD 2 but through two different levels. Note that this feature cannot occur in the simpler model with only two single-level QDs. Summing up, our model reproduces the signatures of nonlocal transport observed in Ref. 31 even though, as we have argued in Sec. IIB, the actual decomposition of the Josephson energy is more complicated than stated in their work.

\section{REGIME OF DOMINANT NONLOCAL TRANSPORT}

Finally, we comment shortly on the regime in which nonlocal transport is dominant, similar to the Cooperpair-splitter regime of superconductor-normal junctions proposed in Ref. 1. In this regime, the superconducting gap is significantly larger than the on-site energies and than the tunnel couplings, $\Delta_{\nu} \gg \varepsilon_{j}, t_{\mu j}$ and excitations in the superconducting leads are highly unfavorable. This suppresses both the cotunneling contributions which allow for a triplet ground state and the sequential Josephson processes which enable a supercurrent to flow in the triplet ground state. As a consequence, there is never a triplet ground state in the limit of large $\Delta_{\nu}$.

When the QDs are brought close to the $(0,0)-(1,1)$ point or to the $(1,1)-(2,2)$ point in the stability diagram, the only remaining transport channel is nonlocal, i.e., the Cooper pairs have to be split: simultaneous transport of

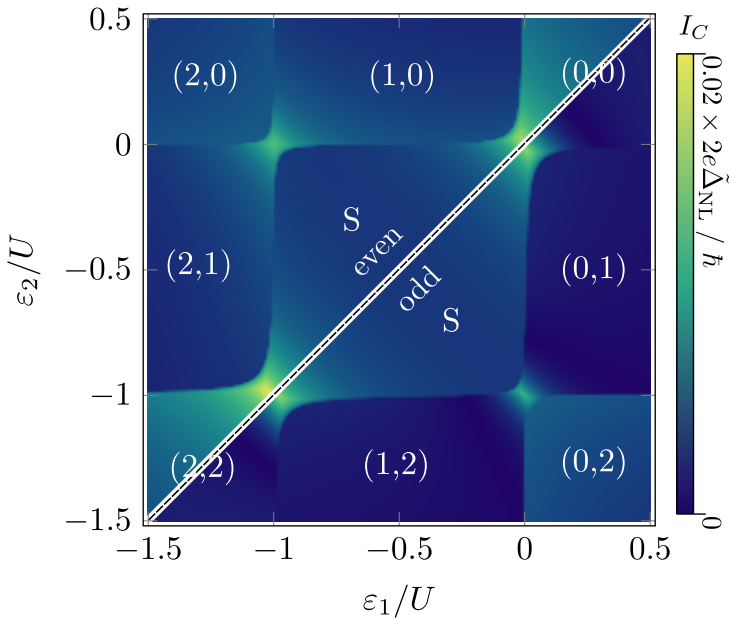

FIG. 7. (Color online) Critical current in the limit of large $\Delta_{\nu}$, where $U_{1}=U_{2} \equiv U, \tilde{\Delta}_{\mathrm{L}}=0.05 U, \tilde{\Delta}_{\mathrm{NL}}=0.025 U$, and $\tilde{t}=0.01 U$. The plot for even and the plot for odd parity are separated by a dashed line. Electrons can leave or enter the superconducting leads only in pairs, so sequential transport is not possible and the single-particle resonances at the groundstate transitions are suppressed. In turn, if the Coulomb repulsion is large, two-particle resonances in the ground state are possible only at four points, where $\varepsilon_{i}=0$ or $\varepsilon_{i}=-U_{i}$ is fulfilled simultaneously for both quantum dots $i=1,2$. At these points, nonlocal transport dominates. Parity has only quantitative influence. In particular, the singlet ground state is stable.

a pair through a single QD is suppressed by the Coulomb repulsion $U_{i}$ and sequential transport of single electrons originating from the same Cooper pair through the QDs is suppressed by the superconducting gap $\Delta_{\nu}$. In this situation, the model can be simplified further by completely integrating out the superconducting leads $\$^{30154}$ to leading order in $t_{\nu i} / \Delta_{\nu}$. Assuming that $\left|\varepsilon_{i}\right|, U_{i}, t_{\nu i} \ll \Delta_{\nu}$ and absorbing a renormalization of the on-site energies into $\varepsilon_{i}$, we obtain

$$
\begin{aligned}
H_{\mathrm{eff}} & =\sum_{i \sigma} \varepsilon_{i} d_{i \sigma}^{\dagger} d_{i \sigma}+\sum_{i} U_{i} d_{i \uparrow}^{\dagger} d_{i \downarrow}^{\dagger} d_{i \uparrow} d_{i \downarrow} \\
& +\sum_{i j \sigma \nu} \tilde{\Delta}_{i j} \mathcal{P}_{\nu} e^{-i \varphi_{\nu}} \sigma d_{i \sigma}^{\dagger} d_{j \bar{\sigma}}^{\dagger}+\text { H.c. } \\
& +\sum_{i j \sigma \nu} \tilde{t} \mathcal{P}_{\nu} d_{i \sigma}^{\dagger} d_{j \sigma},
\end{aligned}
$$

where $\tilde{\Delta}_{i j}$ is the effective amplitude to inject a local $(i=$ $j)$ or a nonlocal $(i \neq j)$ Cooper pair and $\tilde{t}$ describes cotunneling. The parity enters via $\mathcal{P}_{\nu}=1$ if $\nu=L$ and $\mathcal{P}_{\nu}=\mathcal{P}$ if $\nu=R$. Because there are no sequential Cooper-pair transport processes as shown in Fig. 5(b), the triplet sector cannot support any supercurrent and decouples completely. We obtain the critical current in the limit of large $\Delta_{\nu}$ by exact diagonalization of Eq. (15) (Fig. 7).

At a first glance, the behavior of the critical current 
looks deceptively similar to the results discussed before with resonancelike features along the ground-state transitions. But sequential transport is not possible without intermediate excitations in the superconducting leads. So in the $(1,1)$ ground state, transport is possible only in resonance with the $(0,0)$ state, which requires $\varepsilon_{1}+\varepsilon_{2}=0$, and in resonance with the $(2,2)$ state, which requires $\varepsilon_{1}+\varepsilon_{2}=2\left(\varepsilon_{1}+\varepsilon_{2}\right)+U_{1}+U_{2}$. The ground state, however, is $(1,1)$ only if $\varepsilon_{i}<0$ and $\varepsilon_{i}<2 \varepsilon_{i}+U_{i}$. This rules out all configurations except $\varepsilon_{1}=\varepsilon_{2}=0$ and $\varepsilon_{i}=-U_{i}$. Two more nonlocal resonances are found similarly in the sector of odd total occupation when $\varepsilon_{1,2}=0$ and simultaneously $\varepsilon_{2,1}=-U_{2,1}$.

Local transport, on the other hand, is resonant if the $\left(N_{1}, 0\right)$ state is in resonance with the $\left(N_{1}, 2\right)$ state or if the $\left(0, N_{2}\right)$ state is resonant with the $\left(2, N_{2}\right)$ state. In any case, $0=2 \varepsilon_{i}+U_{i}$ is required. But because $U_{i}>0$, this condition is incompatible with any ground state in which QD $i$ is either empty or doubly occupied. Hence there is only nonlocal supercurrent carried by the ground state. Note that the argument is valid only if the resonances are sharp compared to the level spacing, i.e., if the Coulomb repulsion $U_{i}$ is sufficiently large.

This gives a relatively straight-forward signature of nonlocal transport: localized resonances in the $\varepsilon_{1}-\varepsilon_{2}$ plane indicate nonlocal transport. If, on the other hand, only extended steplike features are visible, they are most likely attributable to ground-state transitions and there is no nonlocal Cooper-pair transport.

\section{CONCLUSION}

In this work, we have considered the setup of a doublequantum-dot Josephson junction. We have used exact diagonalization and perturbation theory in the tunneling from the quantum dots to the superconducting leads in order to calculate the critical current of the junction. We included all possible occupations of the quantum-dot levels for various values of the quantum-dot level energies including finite on-site Coulomb repulsion. Depending on the parity of the quantum-dot levels, we discovered a nonlocal (one electron per quantum dot) singlettriplet ground-state transition (at total even parity) as a function of the quantum-dot level energies for a large parameter window when the superconducting gaps are smaller than or comparable to the quantum-dot energy scale. This transition becomes visible as a kink in the critical current with an associated asymmetric line shape between resonances, which could serve as a new sign of coherent Cooper-pair splitting. We consistently find this physics in zero-bandwidth approximation, where each superconducting lead is modeled as a single site with pairing interaction, as well as in the wide-band limit of continuous open leads. Regarding recent experiments 31 on this setup, we included an additional level with opposite parity on one of the quantum dots. We observed critical current traces by varying the gate voltage of the quantum dot with two levels for different but fixed level energies of the other quantum dot that are consistent with the experiment: the different traces are not just shifted by a constant offset but show enhanced relative current profiles near resonances, which, in our model, is directly related to coherent Cooper-pair transport via different quantum dots as was conjectured in Ref. 31 . In addition, we observe asymmetric line shapes between a pair of subsequent resonances due to nonlocal singlettriplet ground-state transitions associated with quantumdot levels showing even total parity. Such asymmetries are also visible in the experiment. Finally, we analyze the model in the limit of large superconducting gaps, where we can integrate out the superconductors thereby creating a proximity effect in the quantum dots. The resulting effective model for the quantum dots can be solved exactly and we find that all current resonances are dominated by nonlocal processes in this limit.

\section{ACKNOWLEDGMENTS}

We gratefully acknowledge discussions with M.-S. Choi and R. S. Deacon, helpful comments on the manuscript by B. Trauzettel and financial support by the EU-FP7 Project SE2ND, No. 271554, Spanish Mineco through grant FIS2014-55486-P, the DFG, Grant No. RE 2978/11 and Research Training Group GrK1952/1 "Metrology for Complex Nanosystems", and the Braunschweig International Graduate School of Metrology B-IGSM. ALY acknowledges financial support from the Spanish MINECO, through the "María de Maeztu" Programme for Units of Excellence in R\&D (MDM-2014-0377).

\section{Appendix A: Diagrammatic perturbation theory}

The correction of the ground-state energy can be obtained conveniently using the Schrieffer-Wolff transformation, which decouples the low-energy degrees of freedom, i.e., in our case the (possibly degenerate) ground state $m$, from the high-energy degrees of freedom, i.e., all excited states $l$, up to fourth order in the tunneling between the QDs and the superconducting leads. Following Ref. 55, the fourth-order contribution to the resulting low-energy Hamiltonian matrix is

$$
\begin{gathered}
H_{m m^{\prime}}^{(4)}=-\frac{1}{2} \sum_{l, l^{\prime}, m^{\prime \prime}} H_{m l} H_{l m^{\prime \prime}} H_{m^{\prime \prime} l^{\prime}} H_{l^{\prime} m^{\prime}} \\
\left(\frac{1}{\left(E_{m}-E_{l}\right)^{2}\left(E_{m}-E_{l^{\prime}}\right)}+\frac{1}{\left(E_{m}-E_{l}\right)\left(E_{m}-E_{l^{\prime}}\right)^{2}}\right) \\
+\sum_{l, l^{\prime}, l^{\prime \prime}} H_{m l} H_{l l^{\prime}} H_{l^{\prime} l^{\prime \prime}} H_{l^{\prime \prime} m^{\prime}} \\
\frac{1}{E_{m}-E_{l}} \frac{1}{E_{m}-E_{l^{\prime}}} \frac{1}{E_{m}-E_{l^{\prime \prime}}}, \quad \text { A }
\end{gathered}
$$


where $E_{\eta}$ is the energy of the unperturbed state $\eta$ and $H_{i j}$ is the matrix element for transitions between states $i$ and $j$. If the unperturbed ground state is unique, $H^{(4)}$ is one dimensional and equivalent to the ground state energy correction $\delta E_{0}$. In the degenerate $(1,1)$-charge sector with $S_{z}=0, H^{(4)}$ is two dimensional. For the calculation of the matrix elements it is convenient to choose as basis $|\uparrow, \downarrow\rangle$ and $|\downarrow, \uparrow\rangle$, such that the absolute value of the offdiagonal terms $H_{12}^{(4)}=H_{21}^{(4) *}$ is half of the singlet-triplet splitting $E_{\mathrm{CT}, \mathrm{se}}^{\mathrm{nl}}+E_{J, \mathrm{se}}^{\mathrm{nl}}(\Delta \varphi)$.

To organize all processes, we represent them by diagrams. We take the point of view of the DQD system. From this point of view, the DQD emits electrons to the leads or absorbs electrons from the leads. Due to the excitation gap of the superconductors, tunneling proceeds in pairs: the DQD can emit an electron into a superconductor which is later reabsorbed, absorb an electron from the Fermi sea and subsequently fill the hole which was created, emit two electrons which form a Cooper pair, or absorb two electrons by destroying a Cooper pair. At this point it does not matter which one of the leads enables the process as later on all possibilities are summed over. To keep track of which QD is affected by one tunneling event, we represent each QD by one horizontal line. Each tunnel event involving the QD is a vertex on this line. A line connecting two vertices indicates, which two tunnel events are connected by one of the processes mentioned above. We name it a lead line. Two example diagrams are shown in Fig. 8 . The process on the DQD is the same but it is mediated by two different lead processes; in Fig. 8(a) the process is mediated by Josephson processes whereas it is mediated by cotunneling in Fig. 8(b). The direction of the arrows on the lead lines indicates the flow of electrons onto or out of the QDs. So the lead lines of Josephson processes have two arrows and cotunneling processes have one arrow. The intermediate DQD occupations are given by numbers or by small spin arrows.

Since all processes conserve the total charge of the DQD, they are always a sequence of two creation and two anihilation events both in the DQD and in the leads. It can easily be checked that all possible sequences decompose into a part concerning the leads and a part concerning the DQD without acquiring an overall fermionexchange sign. But within both of the subsystems, we need to account for possible signs due to fermion exchange. To determine the sign of the QD subsystem, the number of permutations is counted which would be required to arrange all vertices of $\mathrm{QD} 1$ to the left of all vertices of QD 2. If the number is odd, a fermionexchange sign results.

If the spin of the electron on a QD is changed in a spinexchange process, another sign may occur. Changing the spin of a QD can be done either by removing the electron and filling the QD with an electron of opposite spin (intermediate occupation number 0 ) or by adding another electron and removing the first electron afterwards (intermediate occupation number 2). In the second case, an additional exchange of fermions is necessary when re- (a)

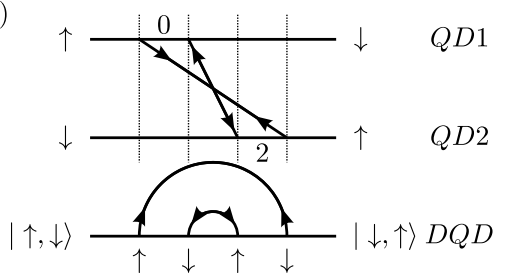

(b)

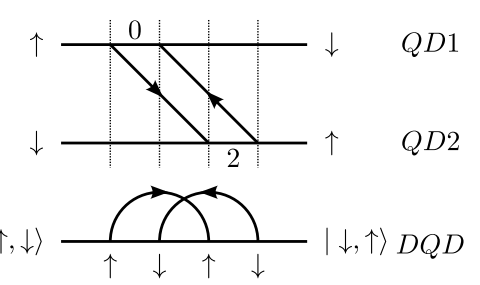

FIG. 8. Example diagram of (a) a spin-exchange Josephson and (b) a spin-exchange cotunneling process with the same intermediate QD occupations (denoted by 0 and 2 electrons) read from the left to the right. The upper two horizontal lines represent the QDs and the lower horizontal line represents the DQD as a whole. The spin arrows at the beginning and at the end of the horizontal lines denote the initial state and the final state of the DQD and the arrows on the lead lines indicate the direction of the electrons flowing out of or into the DQD.

moving the first electron, which we call spin flip via a local singlet. Such a spin flip introduces a sign.

To determine the sign of the processes on the DQD, we can thus summarize the following rules:

- Draw the diagram.

- Count the number of permutations which would be required to arrange all vertices of $\mathrm{QD} 1$ to the left of all vertices of QD 2. If it is odd, add a sign.

- Count the spin flips via a local singlet (intermediate occupation number 2). Each contributes a fermionexchange sign.

To determine the contributions due to the lead process, we construct an auxiliary diagram by collapsing the two lines of the QDs onto one. These auxiliary diagrams are the third horizontal line in Figs. 8 $(a, b)$. Now each crossing of lead lines corresponds to a commutation of lead operators. Furthermore, each lead line represents a normal or an anomalous superconducting correlation function. If, e.g., a lead line connects two events in which, read from the left to the right, first a spin-up electron is removed from the superconductors and then a spin-down electron is removed from the superconductors, the corresponding correlation function is $\left\langle c_{\downarrow} c_{\uparrow}\right\rangle$. All correlation functions can be calculated using the standard Bogoli- 

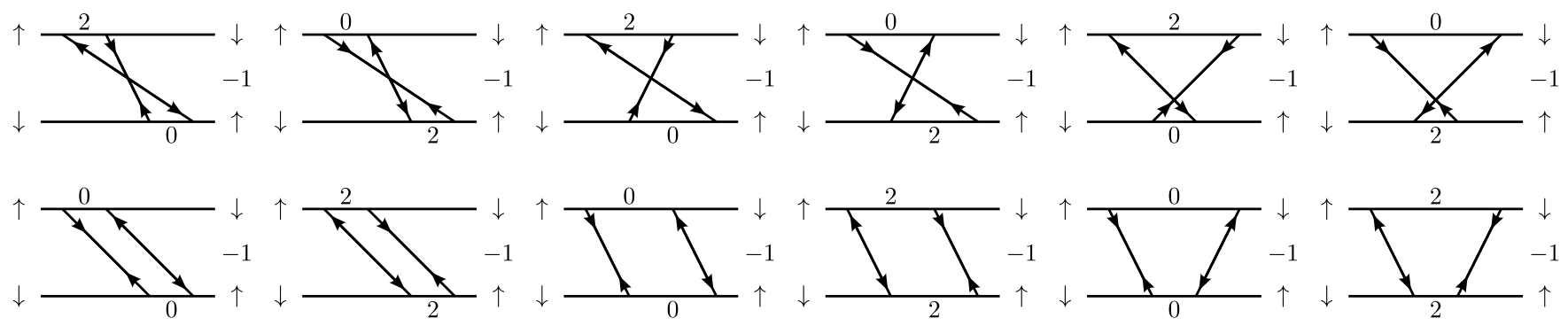

(a)

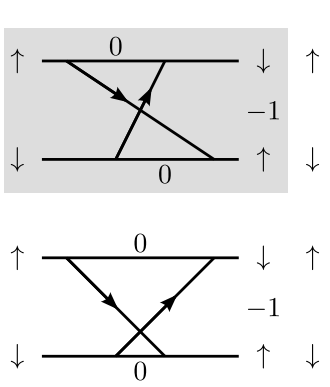

(b)

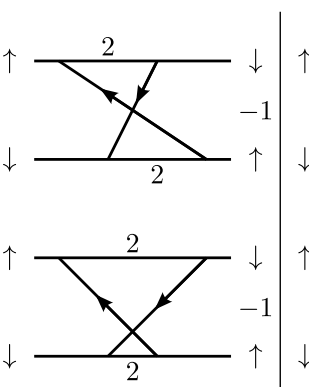

(c)

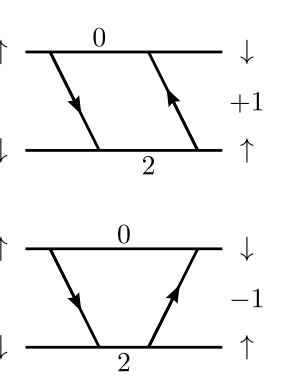

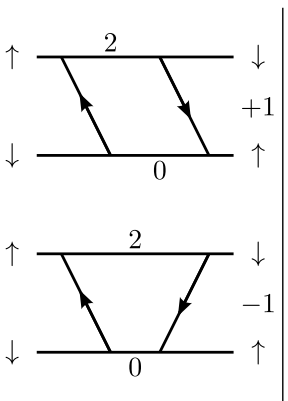

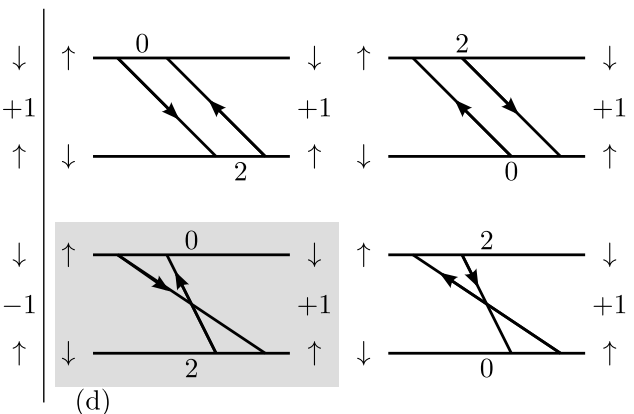

FIG. 9. All diagrams contributing to (a) $\mathcal{M}_{J, \text { se }}^{\mathrm{nl}}$ and (b)-(d) $\mathcal{M}_{\mathrm{CT}, \mathrm{se}}^{\mathrm{nl}}$. (a) All Josephson processes have a negative overall sign and lower the singlet. (b) Cotunneling processes which lower the singlet. The process of Fig. 4(a) is highlighted. (c) Higher orders of second order cotunneling processes come with different signs but cannot lower the triplet since they are contained in the limit of large $\Delta$. (d) Cotunneling processes which lower the triplet. The process of Fig. 4(b) is highlighted.

ubov transform 56

$$
\begin{aligned}
& \left\langle c_{\uparrow}^{\dagger} c_{\downarrow}^{\dagger}\right\rangle=\left\langle c_{\downarrow} c_{\uparrow}\right\rangle=-\left\langle c_{\downarrow}^{\dagger} c_{\uparrow}^{\dagger}\right\rangle=-\left\langle c_{\uparrow} c_{\downarrow}\right\rangle=\frac{\Delta}{2 \sqrt{\varepsilon^{2}+\Delta^{2}}} \\
& \left\langle c_{\uparrow}^{\dagger} c_{\uparrow}\right\rangle=\left\langle c_{\downarrow}^{\dagger} c_{\downarrow}\right\rangle=\frac{1}{2}\left(1-\frac{\varepsilon}{\sqrt{\varepsilon^{2}+\Delta^{2}}}\right) \\
& \left\langle c_{\uparrow} c_{\uparrow}^{\dagger}\right\rangle=\left\langle c_{\downarrow} c_{\downarrow}^{\dagger}\right\rangle=\frac{1}{2}\left(1+\frac{\varepsilon}{\sqrt{\varepsilon^{2}+\Delta^{2}}}\right),
\end{aligned}
$$

where $\varepsilon$ is the normal-state energy of the lead electron measured from the Fermi level. With dispersionful leads such as in the wide-band limit, $\varepsilon$ depends on an internal quantum number, e.g., $\varepsilon_{\mathbf{k}}$, which is summed over. Note that the order of the spins in the superconducting correlation functions is important since the order in which the electrons are put into the Cooper pair condensate matters.

The lead part of the matrix element can thus be obtained by following these rules:

- Collapse the two-line diagram to the auxiliary diagram.

- Count the number of line crossings. Each crossing contributes a fermion-exchange sign.

- Write down the lead correlations following Eq. A2. Take care of fermion-exchange signs that might occur due to the spin-order of Cooper pairs. Use $\varepsilon$ for one lead line and $\varepsilon^{\prime}$ for the other line.

Finally, we need to determine the energies of the virtual states. The corresponding energies of the DQD can be read off from the two-line diagram. The energies of the three virtual states can be found by looking at the states in the three spaces between the dashed lines in the two line diagrams. Each lead line contributes an additional energy $\sqrt{\varepsilon^{2}+\Delta^{2}}$ and $\sqrt{\varepsilon^{\prime 2}+\Delta^{2}}$, for the two pairs of tunneling events respectively.

By drawing all diagrams and inserting the corresponding matrix elements and the energies into Eq. (A1), the fourth-order corrections of the ground state energy can be constructed explicitly. All of the diagrams of the spinexchange contributions $\mathcal{M}_{\mathrm{CT} / J \text {,se }}^{\mathrm{nl}}$ are shown in Fig. 9. All of them are nonlocal since the lead lines connect the two QD lines. From the overall exchange signs given next to the diagrams we see that all of the Josephson processes [Fig. 9(a)] and some of the genuine fourth-order cotunneling processes [Fig. 9.(b)] lower the singlet ground state. The processes which are second order in electron cotunneling or Cooper pair tunneling correspond to diagrams which are reducible in the sense that they can be cut into two parts vertically without cutting a lead line [Fig. 9(c)]. They are the processes surviving the limit of large $\Delta$ (Sec. $V$ ) and hence they lower the singlet ground state. The remaining cotunneling processes lower the triplet ground state [Fig. $9(\mathrm{~d})]$. 
1 P. Recher, E. V. Sukhorukov, and D. Loss, Phys. Rev. B 63, $165314(2001)$

${ }^{2}$ G. Lesovik, T. Martin, and G. Blatter, Eur. Phys. J. B 24, 287 (2001)

${ }^{3}$ P. Recher and D. Loss, Phys. Rev. B 65, 165327 (2002)

${ }^{4}$ C. Bena, S. Vishveshwara, L. Balents, and M. P. A. Fisher, Phys. Rev. Lett. 89, 037901 (2002).

5 P. Recher and D. Loss, Phys. Rev. Lett. 91, 267003 (2003)

${ }^{6}$ E. Prada and F. Sols, Eur. Phys. J. B 40, 379 (2004)

7 W. D. Oliver, F. Yamaguchi, and Y. Yamamoto, Phys. Rev. Lett. 88, 037901 (2002),

$\therefore$ A. Levy Yeyati, F. S. Bergeret, A. Martin-Rodero, and T. M. Klapwijk, Nat. Phys. 3, 455 (2007).

9 J. Cayssol, Phys. Rev. Lett. 100, 147001 (2008)

${ }^{10}$ K. Sato, D. Loss, and Y. Tserkovnyak, Phys. Rev. Lett. 105, $226401(2010)$

i1 A. Schroer, P. G. Silvestrov, and P. Recher, Phys. Rev. B 92, $241404(2015)$

${ }^{12}$ L. Hofstetter, S. Csonka, J. Nygård, and C. Schönenberger, Nature (London) 461, 960 (2009)

13 L. G. Herrmann, F. Portier, P. Roche, A. Levy Yeyati, T. Kontos, and C. Strunk, Phys. Rev. Lett. 104, 026801 (2010)

14 A. Das, Y. Ronen, M. Heiblum, D. Mahalu, A. V. Kretinin, and H. Shtrikman, Nat. Commun. 3, 1165 (2012).

15 Z. B. Tan, D. Cox, T. Nieminen, P. Lähteenmäki, D. Golubev, G. B. Lesovik, and P. J. Hakonen, Phys. Rev. Lett. 114, $096602(2015)$

10 G. Fülöp, F. Domínguez, S. d'Hollosy, A. Baumgartner, P. Makk, M. H. Madsen, V. A. Guzenko, J. Nygård, C. Schönenberger, A. Levy Yeyati, and S. Csonka, Phys. Rev. Lett. 115, 227003 (2015)

1 S. Kawabata, J. Phys. Soc. Jpn. 70, 1210 (2001)

18 N. M. Chtchelkatchev, G. Blatter, G. B. Lesovik, and T. Martin, Phys. Rev. B 66, 161320 (2002).

19 B. Braunecker, P. Burset, and A. Levy Yeyati, Phys. Rev. Lett. 111, 136806 (2013)

${ }^{20}$ G. Burkard, D. Loss, and E. V. Sukhorukov, Phys. Rev. B 61, R16303 (2000)

${ }^{21}$ X. Hu and S. Das Sarma, Phys. Rev. B 69, 115312 (2004).

22 P. Samuelsson, E. V. Sukhorukov, and M. Büttiker, $\overline{\text { Phys. }}$ Rev. B 70, 115330 (2004).

23 P. San-Jose and E. Prada, Phys. Rev. B 74, 045305 (2006)

24 F. Mazza, B. Braunecker, P. Recher, and A. Levy Yeyati, Phys. Rev. B 88, 195403 (2013).

25 A. Schroer, B. Braunecker, A. Levy Yeyati, and P. Recher, Phys. Rev. Lett. 113, 266401 (2014)

26 V. Cerletti, O. Gywat, and D. Loss, Phys. Rev. B 72, 115316 (2005)

27 M. Titov, B. Trauzettel, B. Michaelis, and C. W. J. Beenakker, New J. Phys. 7, 186 (2005).

28 J. C. Budich and B. Trauzettel, Nanotechnology 21, 274001 (2010).

${ }^{29}$ S. E. Nigg, R. P. Tiwari, S. Walter, and T. L. Schmidt, Phys. Rev. B 91, 094516 (2015)

30 A. Schroer and P. Recher, Phys. Rev. B 92, 054514 (2015).

31 R. S. Deacon, A. Oiwa, J. Sailer, S. Baba, Y. Kanai, K. Shibata, K. Hirakawa, and S. Tarucha, Nat. Commun. 6, 7446 (2015)

32 M.-S. Choi, C. Bruder, and D. Loss, Phys. Rev. B 62, 13569 (2000)
33 Z. Wang and X. Hu, Phys. Rev. Lett. 106, 037002 (2011).

${ }^{34}$ H. Pan and T.-H. Lin, Phys. Rev. B 74, 235312 (2006)

35 R. Jacquet, J. Rech, T. Jonckheere, A. Zazunov, and T. Martin, Phys. Rev. B 92, 235429 (2015).

${ }^{36}$ M. Lee, T. Jonckheere, and T. Martin, Phys. Rev. B 81, $155114(2010)$

37 A. V. Rozhkov and D. P. Arovas, Phys. Rev. Lett. 82, 2788 (1999)

38 F. S. Bergeret, A. L. Yeyati, and A. Martín-Rodero, Phys. Rev. B 74, 132505 (2006).

${ }^{39}$ R. López, M.-S. Choi, and R. Aguado, Phys. Rev. B 75, 045132 (2007)

${ }^{40}$ H. R. Krishna-murthy, J. W. Wilkins, and K. G. Wilson, Phys. Rev. B 21, 1003 (1980).

41 M.-S. Choi, M. Lee, K. Kang, and W. Belzig, Phys. Rev. B 70, 020502 (2004)

42 M. Governale, M. G. Pala, and J. König, Phys. Rev. B $\mathbf{7 7}, 134513(2008)$

${ }^{43}$ F. Siano and R. Egger, Phys. Rev. Lett. 93, 047002 (2004).

44 A. A. Clerk and V. Ambegaokar, Phys. Rev. B 61, 9109 (2000)

45 I. Affleck, J.-S. Caux, and A. M. Zagoskin, Phys. Rev. B 62, $1433(2000)$

${ }^{40}$ E. Vecino, A. Martín-Rodero, and A. L. Yeyati, Phys. Rev. B 68, 035105 (2003).

47 F. S. Bergeret, A. L. Yeyati, and A. Martín-Rodero, Phys. Rev. B 76, 174510 (2007).

48 As the charging state of the QDs must not change, Josephson processes and cotunneling processes do not mix to fourth order, cf. App. A.

49 An important reason that the critical current differs between the singlet phase and the triplet phase is the sign of the nonlocal Josephson current. As can be seen from Eqs. 9) and (10), it depends on the phase, on the parity, and on whether the ground state is a singlet or a triplet. So if the local supercurrents and the nonlocal supercurrent are flowing in opposite directions at $\Delta \varphi= \pm \pi / 2$, it can be beneficial to switch to the other ground state at $\Delta \varphi^{\prime} \neq \pm \pi / 2$, where the individual supercurrents are smaller but flow in the same direction. Due to this interplay it is nontrivial to isolate nonlocal features from the critical current.

50 Substituting the ground-state energy with the free energy, $I=-2 e k T / \hbar \times \partial_{\Delta \varphi} \ln \sum_{n} e^{-\beta E_{n}, 57}$ we verify that the total-spin transition is present also at finite temperatures, $k T \sim 0.1 \Delta$, and hence experimentally accessible.

51 When integrating out the momentum quantum number in the case of continuous leads, at large $\Delta$, second-order Cooper-pair processes are independent of $\Delta$ so the relative suppression of genuine fourth-order processes may be even stronger.

${ }^{52}$ It is helpful to consider the equivalent behavior of the fullypolarized triplet.

53 Here, we neglect the spin-exchange interaction within QD 2. Adding it, however, would not change our results because the device is in the single-level regime, $\delta \gg U_{2 i}, t$.

${ }^{54}$ P. Recher, Y. V. Nazarov, and L. P. Kouwenhoven, Phys. Rev. Lett. 104, 156802 (2010).

5o R. Winkler, Spin-orbit coupling effects in two-dimensional electron and hole systems (Springer, Berlin, 2003).

56 M. Tinkham, Introduction to superconductivity, 2nd ed. (McGraw-Hill, New York, 1996). 
${ }^{57}$ H. Bruus and K. Flensberg, Many-Body Quantum Theory in Condensed Matter Physics (Oxford University Press, 2004). 